\section{RMD Open}

Rheumatic \&

Musculoskeletal Diseases

\title{
Part of pain labelled neuropathic in rheumatic disease might be rather nociplastic
}

Florian Bailly (D, ${ }^{1,2}$ Alain Cantagrel, ${ }^{3}$ Philippe Bertin, ${ }^{4}$ Serge Perrot, ${ }^{5,6}$

Thierry Thomas, ${ }^{7}$ Thibaud Lansaman, ${ }^{8}$ Laurent Grange, ${ }^{9}$ Daniel Wendling (D), ${ }^{10}$ Calogera Dovico, ${ }^{11}$ Anne-Priscille Trouvin (10),12

To cite: Bailly F, Cantagrel A, Bertin $\mathrm{P}$, et al. Part of pain labelled neuropathic in rheumatic disease might be rather nociplastic. RMD Open 2020;6:e001326. doi:10.1136/ rmdopen-2020-001326

- Prepublication history and additional material for this paper are available online. To view these files, please visit the journal online (http://dx.doi. org/10.1136/rmdopen-2020001326).

Received 14 May 2020 Revised 23 July 2020 Accepted 21 August 2020
Check for updates

\section{(c) Author(s) (or their} employer(s)) 2020. Re-use permitted under CC BY-NC. No commercial re-use. See rights and permissions. Published by BMJ.

For numbered affiliations see end of article.

\section{Correspondence to}

Florian Bailly;

florian.bailly@aphp.fr

\section{ABSTRACT}

Pain in rheumatic diseases is primarily due to mechanical or inflammatory mechanism, but neuropathic pain (NP) component is also occurring in many conditions and is probably underdiagnosed. The purpose of this article is to provide an overview of prevalence, pathophysiological and currently available treatment of NP in rheumatic diseases. When associated with clinical evaluation assessing neurological clinical signs and neuroanatomical distribution, Douleur Neuropathique 4 Questions, painDETECT, Leeds assessment of neuropathic symptoms and signs and Neuropathic Pain Questionnaire can detect NP component. Inflammatory or connective diseases, osteoarthritis, back pain or persistent pain after surgery are aetiologies that all may have a neuropathic component. Unlike nociceptive pain, NP does not respond to usual analgesics such as paracetamol and non-steroidal anti-inflammatory drugs. Entrapment neuropathy, peripheral neuropathy or smallfibre neuropathy are different aetiologies that can lead to NP. A part of the pain labelled neuropathic is rather nociplastic, secondary to a central sensitisation mechanism. Identifying the right component of pain (nociceptive vs neuropathic or nociplastic) could help to better manage pain in rheumatic diseases with pharmacological and nonpharmacological treatments.

\section{INTRODUCTION}

According to the International Association for the Study of Pain (IASP), the current definition of neuropathic pain (NP) is 'Pain caused by a lesion or disease of the somatosensory nervous system'. ${ }^{1} \mathrm{NP}$ is a clinical description (and not a diagnosis), which requires a demonstrable lesion or a disease that satisfies established neurological diagnostic criteria. The term lesion is commonly used when diagnostic investigations (eg, imaging, neurophysiology, biopsies, laboratory tests) reveal an abnormality or when there is an obvious trauma history. The term disease is commonly used when the underlying cause of the lesion is known (eg, stroke, vasculitis, diabetes

\section{Key messages}

Prevalence of neuropathic or nociplastic pain component in rheumatic diseases ranges from $3 \%$ to $50 \%$ depending on the pathology.

- A part of the pain labelled neuropathic is rather nociplastic, secondary to a central sensitisation mechanism.

- Clinical characteristics and questionnaires that can be used to identify and better manage neuropathic or nociplastic pain are described.

mellitus, genetic abnormality). Somatosensory refers to information about the body per se including visceral organs, rather than information about the external world (eg, vision, hearing or olfaction). The presence of symptoms (burning sensation) or signs (eg, touchevoked pain) alone does not justify the use of the term neuropathic.

NP occurs in cases of injury or disease of the somatosensory nervous system, but the mechanisms involved in its maintenance also include microglia and astrocyte activation of the spinal cord, by promoting local inflammation in the dorsal horn of the spinal cord. ${ }^{2}$ A peripheral nerve damage results in releasing powerful neuromodulators such as proinflammatory cytokines and chemokines, which modify the transduction of the nervous signal to the central nervous system. This inflammation could create maladaptive synaptic circuits and activate intracellular signalling events that permanently contribute to enhanced NP. ${ }^{3}$

Prevalence of NP in general population is partially known: in 2008, a large nationwide postal survey in France among 30155 subjects found a $6.9 \%$ prevalence of chronic pain with neuropathic characteristics. ${ }^{4}$ Prevalence of moderate or severe pain with neuropathic 
characteristics was $5.1 \%$ in this study. A systematic review of NP in the general population published in 2014 came to the conclusion that a better estimation of population prevalence of pain with neuropathic characteristics is likely to range between $6.9 \%$ and $10 \% .^{5}$

A subgroup of patients with different types of pain, with clinical features suggestive of NP component, has been identified in many rheumatological pathologies. For example, in osteoarthritis (OA), some patients experience altered proprioception or cutaneous vibration sensitivity, hypoaesthesia, correlated with the intensity of pain but not with radiographic changes. ${ }^{6}$ These abnormalities, usually related to NP, are present at the painful joint, but also at a remote location, suggesting that the loss of function is mediated centrally and not peripherally. ${ }^{6}$ These observations suggested that a proportion of patients with rheumatic disease could have mixed, not just nociceptive pain, even though no 'damage' to the nervous system was identified.

In the 11th revision of the International Classification of Disease, NP and musculoskeletal pain are two distinct entities. $^{78}$ In some diseases, both can coexist, and the expression 'NP component' or 'mixed pain' is sometimes used to differentiate with nociceptive pain. Yet this term is frequently used because the new terminology of 'nociplastic pain', which is different from NP, is not well known. This could lead to an overestimation of NP component in patients with rheumatic diseases.

It is important to differentiate between patients with purely nociceptive pain and those with neuropathic or nociplastic pain, because the usual pain treatments (analgesics, anti-inflammatory drugs) are less effective for neuropathic or nociplastic pain, ${ }^{9}$ while specific treatments such as tricyclic antidepressants can be effective. NP component is also an independent prognostic factor for knee prosthetic replacement failure.$^{10}$ Identifying subgroups of patients with NP component would allow a better management of these patients. The purpose of this article is to provide an overview of prevalence, pathophysiological and currently available treatment of neuropathic and nociplastic pain component in rheumatic diseases.

\section{DIAGNOSIS OF NP}

Examination of a patient presenting with pain starts with interviewing the patient about his or her symptoms (onset, location, intensity, associated diseases). ${ }^{11}$ The severity of pain and its impact on daily life, including disability and effect on sleep and mood, should be explored. NP screening tools can be used to alert the physician to the possibility of NP. The main tools used are as follows:

- DN4 (Douleur Neuropathique 4 Questions) consists of seven items related to symptoms and three other items related to clinical examination. ${ }^{12}$ The DN4 is easy to score, and a total score of 4 of 10 or higher suggests NP. The seven sensory descriptors can be used as a selfreport questionnaire with similar results. The questionnaire was developed and validated in French and has been translated into 15 languages.

- PainDETECT questionnaire (PDQ) was developed and validated in German ${ }^{13}$ and is available in 22 languages, including French. It incorporates a self-report questionnaire with nine items that do not require a clinical examination. There are seven weighted sensory descriptor items and two items related to the spatial (radiating) and temporal characteristics of the individual pain pattern. A neuropathic component is unlikely if score $\leq 12$, likely if score $\geq 19$ and uncertain between these two values.

- The Leeds assessment of neuropathic symptoms and signs (LANSS) contains five symptom items and two clinical examination items. ${ }^{14}$ A score of 12 or higher (out of a possible 24) suggests NP. A self-report tool, the Self-Reported LANSS, has also been validated. After the initial validation study, the LANSS has been tested and validated in several settings with sensitivity and specificity ranging from $82 \%$ to $91 \%$ and $80 \%$ to $94 \%$, respectively, compared to clinical diagnosis.

The DN4, LANSS and PDQ are those most frequently used in clinical practice. The Neuropathic Pain Symptom Inventory is less used for screening but is sometimes useful for follow-up, as it allows the individualisation of five subcomponents of NP: spontaneous burning pain, spontaneous deep pain, paroxysmal pain, evoked pain and paraesthesia/dysaesthesia. $^{15} 16$ Many other questionnaires exist, such as the Neuropathic Pain Questionnaire (NPQ) which consists of 12 items, 10 of which are related to sensations or sensory responses, and the other 2 related to affect ${ }^{17}$ but is more frequently used in research. ${ }^{15}$

Those tools can detect NP component but cannot make a definite diagnosis. A grading system to identify possible, probable or definite NP was published. ${ }^{17}$ The algorithm highlighted the need to evaluate if the pain distribution was neuroanatomically possible and if history suggested relevant lesion or disease. A definite NP requires sensory signs confined to innervation territory of the damaged nervous structure together with diagnostic test confirming lesion or disease which can plausibly explain NP. It is common practice for laboratory evaluations (electromyography, evoked potentials, skin biopsy) to be mandatory if the history and symptoms are compatible with NP, but clinical findings remain normal or equivocal. ${ }^{11}$

Only two of those questionnaires (DN4 and LANSS) included not only patient evaluation but also physical examination: hypoesthesia and allodynia. However, the presence of an abnormality in the physical examination is not required to achieve the diagnostic score. An evaluation of patients with fibromyalgia and diabetic neuropathy showed that the two pains were different but that a questionnaire could not differentiate between them. ${ }^{18}$

\section{MECHANISM OF NP COMPONENT}

Multiple mechanisms can be involved in NP components in rheumatic diseases. 
- Entrapment neuropathy can occur in rheumatic inflammatory diseases, especially at the beginning of the disease. The median nerve can be entrapped at the wrist level to cause carpal tunnel syndrome. The ulnar nerve can be entrapped at the elbow to cause cubital tunnel syndrome and at the wrist to cause Guyon's canal syndrome. ${ }^{19}$ The most frequent entrapment neuropathy is carpal tunnel syndrome, which is sometimes secondary to inflammation of the sheath of flexor tendons. Inflammatory rheumatism such as rheumatoid arthritis (RA) ${ }^{20}$ must be sought.

- In RA, spinal cord compression can also be caused by cervical spine disorders such as atlantoaxial dislocation. ${ }^{19}$

- Peripheral neuropathy may be an extra-articular manifestation of the disease. In systemic lupus erythematosus, the prevalence of peripheral neuropathy reportedly varies from $5 \%$ to $27 \%$ of the patients and is characterised mostly by a length-dependent mild sensory or sensorimotor neuropathy. ${ }^{21}$ In diseases that have been progressing for a long time with significant inflammation, amyloidosis can appear and lead to neuropathy. ${ }^{22}$

- Small-fibre neuropathy is defined as a damage to the peripheral nerves that predominantly or entirely affects the small myelinated (A $\delta$ ) fibres or unmyelinated C fibres. Fifty per cent of the cases of smallfibre neuropathy are idiopathic, but they can be associated with rheumatic diseases such as Sjögren syndrome ${ }^{23}$ or sarcoidosis. ${ }^{24}$ When this pathology is associated with length-dependent neuropathy, such as diabetic neuropathy, the clinical diagnosis is simple, and the neurological disease can be confirmed by an electromyogram. Although when the small-fibre neuropathy is isolated, only specific tests such as laserevoked potentials or a skin biopsy can confirm the diagnosis. Clinical assessment is difficult, because NP does not have a length-dependent topography or is not related to a dermatomal distribution.

\section{DIFFERENCES BETWEEN NEUROPATHIC AND NOCIPLASTIC PAIN}

The new definition of NP no longer includes 'dysfunction of the nervous system', which suggests that components of pain are not only nociceptive or neuropathic. A third mechanism for chronic pain has been proposed: nociplastic pain. ${ }^{25}$ Defined as 'pain that arises from altered nociception despite no clear evidence of actual or threatened tissue damage causing the activation of peripheral nociceptors or evidence for disease or lesion of the somatosensory system causing the pain', ${ }^{26}$ nociplastic pain is linked with different mechanisms, including central sensitisation. Central sensitisation is an increased responsiveness of nociceptive neurons in the central nervous system to their normal or subthreshold afferent input. This enhancement in the function of neurons and circuits in nociceptive pathways is caused by increases in membrane excitability and synaptic efficacy as well as reduced inhibition, secondary to the plasticity of the central nervous system. ${ }^{27}$ This amplification of neural signalling elicits pain hypersensitivity. This does not mean that the pain is not real, just that it is not only activated by noxious stimuli. This mechanism is involved in a large component of pain in multiple conditions: fibromyalgia, OA, musculoskeletal disorders with generalised pain hypersensitivity, headache, back pain, temporomandibular joint disorders, NP, visceral pain hypersensitivity disorders and postsurgical pain. ${ }^{28} \mathrm{How}-$ ever, for some authors, central sensitisation mechanism is preferably related to nociplastic pain and does not have the same pathophysiological mechanism that NP: central sensitisation is defined by the IASP as an 'increased responsiveness of nociceptive neurons in the central nervous system to their normal or subthreshold afferent input', and therefore is not related to a lesion or a disease of the somatosensory system, which is necessary for the diagnosis of NP. ${ }^{826}$

Patients can have a combination of nociceptive and nociplastic pains. This definition is recent, and most articles do not differentiate NP from nociplastic pain yet, especially as some of their clinical characteristics overlap.

All the questionnaires described at the beginning of this article have been developed between 2001 and 2006, when definition of NP included 'lesion or dysfunction of the central nervous system', and nociplastic pain was not clearly individualised. However, the inclusion criteria for patients in whom the questionnaires were validated generally excluded nociplastic pain (called 'dysfunctional' at the time) such as fibromyalgia. ${ }^{15}$

\section{HOW TO ASSESS THE PRESENCE OF NOCIPLASTIC PAIN}

The typical pattern of nociplastic pain is fibromyalgia. Patients with fibromyalgia can be identified using the 2016 revision of fibromyalgia diagnostic criteria. ${ }^{29}$ Generalised pain for at least 3 months, associated with widespread pain index (WPI) $\geq 7$ and symptom severity scale (SSS) score $\geq 5$ OR WPI of 4-6 and SSS score $\geq 9$. It is important to note that the diagnosis can be made even if another painful condition is associated, such as chronic inflammatory rheumatisms or connective tissue diseases.

To help diagnosis of nociplastic pain, the clinician may be helped by investigating associated disorders such as headache, temporomandibular joint dysfunction, digestive or urinary functional disorders, fatigue or mood disorders. These elements are part of the fibromyalgia severity score, whereas they are not usually present in NP. ${ }^{29}$

The Central Sensitisation Inventory is a questionnaire that has been developed to identify the presence of pain of the central sensitisation type, regardless of a specific aetiology, ${ }^{30} 31$ with good psychometric characteristics. ${ }^{32}$ However, some studies evaluate the presence of central sensitisation using NPQ: the Pain DETECT questionnaire is used to evaluate nociplastic pain for patients with OA or RA, and correlated with functional brain connectivity alterations linked with central sensitisation on MRI. ${ }^{33} 34$ Since the clinical features are very similar, it is not always 
possible to differentiate between these two pains. Identifying NP rather than nociplastic pain can be done using the Neuropathic Pain Special Interest Group (NeuPSIG) algorithm: definite NP requires both (1) negative or positive sensory signs, confined to innervation territory of the lesioned nervous structure and (2) diagnostic test confirming lesion or disease explaining NP (using electroneuro-myography or MRI, eg). ${ }^{11}$ The presence of only one of these two characteristics can only indicate probable NP. Conversely, the presence of a diffuse territory of pain orientates towards nociplastic pain. To objectively identify central sensitisation, quantitative sensory testing (QST) is the gold standard..$^{35}$ This standardised method is widely used in research to identify central sensitisation, but the length of time and cost of the material required does not allow it to be used in current practice. In patients with OA, modification of QST is correlated with high PDQ. In summary, the clinical characteristics of neuropathic and nociplastic pain are overlapping and difficult to differentiate only with questionnaires, although their pathophysiological mechanisms are different.

\section{PREVALENCE OF NEUROPATHIC OR NOCIPLASTIC PAIN COMPONENT IN RHEUMATIC DISEASES}

In inflammatory diseases, although pain is traditionally considered to be of peripheral nociceptive origin, NP is much more frequent than in the general population. In a large survey including 7054 patients registered in DANBIO (nationwide registry of biological therapies in Denmark), the presence of NP was investigated using the PDQ in different rheumatic conditions. ${ }^{36}$ In RA, NP features were present in $20 \%$ of the patients. In psoriatic arthritis and other spondyloarthritis, $28 \%$ and $21 \%$ had this feature. In this subset of patients, all patient-reported outcomes were higher with more pain, fatigue and disability, and poorer global health. In contrast, there were no differences in $\mathrm{C}$ reactive protein, serology and current biological treatment. In patients with RA and psoriatic arthritis, higher tender joint counts were observed in the highest PDQ classification group. In patients with other spondyloarthritis, a lower proportion of HLA-B27-positive patients was observed in the highest PDQ classification group. Other studies have found NP in $3-33 \%$ of the patients. ${ }^{37-39}$ Central sensitisation is the main hypothesis to explain these clinical characteristics. ${ }^{37}$ Identifying these patients makes it possible not to intensify their biological treatments (which will most certainly be ineffective in this particular context of pain) and offer them adapted care.

In connective tissue diseases, such as systemic lupus erythematosus, peripheral neuropathy prevalence in a large longitudinal study was $5.9 \%$ and was associated with disease activity. The most common lesion was axonal neuropathy in $56 \%$ of these patients, but small-fibre neuropathy was also involved. Association with Sjögren's disease, a common cause of small-fibre neuropathy, ${ }^{23}$ was not reported.
In OA, several studies have suggested an NP component which was found in 5-34\% of the patients ${ }^{40}$ Pain in OA is probably driven by both structural joint changes and abnormal excitability in peripheral and central pain pathways. A deeper understanding of multiple mechanisms of OA pain has led to the use of centrally acting medicines, which may have a positive impact on alleviating osteoarthritic pain, particularly in patients with other centrally mediated symptoms such as fatigue or mood disturbances. ${ }^{40}$ Nevertheless, the recommendations of the Osteoarthritis Research Society International did not conclude that these treatments are effective for the majority of patients. ${ }^{41}$

Patients with pain and advanced OA may undergo surgery such as total knee or hip arthroplasty, but the presence of neuropathic component was associated with poorer surgical outcome. ${ }^{4243}$

After knee or hip arthroplasty, nerve injury can lead to $\mathrm{NP}$ and is present in approximately $6 \%$ of the patients. ${ }^{44}$ Complex regional pain syndrome can also result in chronic pain with neuropathic component. ${ }^{45}$

In low back pain, large epidemiological studies have shown that $20-35 \%$ of the patients suffer from an NP component. ${ }^{46}$ Radicular pain associated with back pain is the most common NP syndrome, even if other mechanisms may be involved. Sensitisation processes of the peripheral nerves or roots could induce a secondary central sensitisation of spinal cord neurons, which plays an additional role in these abnormal NP conditions.

The prevalence of nociplastic pain, independent of NP, is difficult to determine in these diseases, particularly given the heterogeneity of the methods used and the limited number of studies using the most reliable QST method. ${ }^{37}$

\section{IMPLICATION FOR PAIN MANAGEMENT}

To date, the management of peripheral NP remains essentially symptomatic, and aimed at pain relief and improving the patient's quality of life. Unlike nociceptive pain, NP does not respond to usual analgesics such as paracetamol and non-steroidal anti-inflammatory drugs. ${ }^{47}{ }^{48}$ Regarding NP management, several guidelines have been published, including those from Société Française d'Etude et de Traitement de la Douleur (French Pain Society) in 2010, National Institute of Health and Clinical Excellence (British Pain Society) in 2013 and the NeuPSIG of the IASP in 2015. ${ }^{48-50}$ Numerous pharmacological treatments are proposed for NP, such as tricyclic antidepressants, serotonin-norepinephrine reuptake inhibitors, gabapentinoids, topical agents such as lidocaine or high-concentrated capsaicin patch, botulinum toxin A, tramadol or strong opioids. However, these treatments have mainly been tested on postherpetic or diabetic neuropathy, not specifically for nociplastic pain.

The main treatments proposed for fibromyalgia, which is the nociplastic pain model, have some similarities, but 
not all treatments indicated for NP have been tested or validated for fibromyalgia. EULAR guidelines for fibromyalgia recommend mainly non-pharmacological management. When pharmacological treatments are to be discussed, low-dose amitriptyline, duloxetine or milnacipran, tramadol, pregabalin or cyclobenzaprine are therapeutic options. ${ }^{51}$

\section{CONCLUSION}

Pain is the most common manifestation of rheumatic diseases. The frequency of a neuropathic component in rheumatic diseases is yet to be fully evaluated and described, with a particular attention to the distinction with nociplastic pain. Nociplastic pain and NP overlap regarding their clinical feature and are difficult to differentiate only with questionnaires, although their pathophysiological mechanisms are different. The questionnaires usually carried out in current practice do not make it possible to differentiate NP from nociplastic pain. Only objective elements such as negative or positive sensory signs, confined to innervation territory of the lesioned nervous structure and diagnostic test confirming lesion or disease explaining NP allow to confirm NP. In most other situations, the pain known as NP component may be rather nociplastic pain. Identifying neuropathic or nociplastic pain component in patients with rheumatic diseases is required to manage it and improve patients' quality of life. Indeed the current recommendations of treatment for NP may not entirely be efficient for nociplastic pain. Most of the studies selected in the metanalyses used to validate guidelines for neuropathic pain management are based on diabetic neuropathy or postherpetic neuralgia, and cannot be completely duplicated for nociplastic pain.

\section{Author affiliations}

${ }^{1}$ Pain Department, Pitie-Salpetriere Hospital, Assistance Publique - Hopitaux De Paris, Paris, France

${ }^{2}$ Paris 6 University, GRC-UPMC 08, Pierre Louis Institute of Epidemiology and Public Health, Paris, France

${ }^{3}$ Rheumatology, C.H.U. Purpan, Toulouse, France

${ }^{4}$ Hopital Dupuytren, Limoges, France

${ }^{5}$ Centre d'Evaluation Et Traitement De La Douleur, Université Paris Descartes, Hopital Cochin, Paris, France

${ }^{6}$ U987, INSERM, Boulogne Billancourt, France

${ }^{7}$ Rheumatology, CHU De St-Etienne, Saint-Etienne,France

${ }^{8}$ Médecine Physique Et Réadaptation, Hôpital Raymond-Poincaré - Assistance

Publique Hôpitaux De Paris, Garches, France

${ }^{9} \mathrm{CHU}$ Grenoble, Grenoble, France

${ }^{10}$ Rheumatology, CHRU (University Hospital), Besançon, France

${ }^{11}$ Service De Médecine Physique Et Réadaptation, Dispositif IEM APF FRANCE HANDICAP, Belfort, France

${ }^{12}$ Pain Evaluation and Treatment, Hopital Cochin, Paris, France

Twitter Florian Bailly @baillyflo.

Acknowledgements The authors would like to thank the Grünenthal laboratory for its logistical support.

Contributors All authors have substantially contributed to conducting the underlying research and drafting this manuscript.

Funding The scientific writing of the article was carried out independently of the Grünenthal Laboratories, without financial compensation. Grünenthal Laboratories funded the submission of this manuscript.
Competing interests FB reports personal fees from Grünenthal, during the conduct of the study; personal fees from Lilly, Boston and Novartis, outside the submitted work; AC reports personal fees from Grunhental, outside the submitted work; PB reports personal fees from Grünenthal, during the conduct of the study; SP reports personal fees from Grünenthal, grants and personal fees from Sanofi and Pfizer, during the conduct of the study; $T T$ reports personal fees from Grünenthal, during the conduct of the study; grants and personal fees from Amgen, Chugai, UCB, Pfizer, MSD and Novartis, personal fees from Gilead, Arrow, Biogen, BMS, Expanscience, Gilead, LCA, Lilly, Medac, Nordic, Sandoz, Sanofi, Theramex, Thuasne and TEVA, grants from Bone Therapeutics, outside the submitted work; TL reports personal fees from Grunenthal, Allergan, IPSEN and MERZ, outside the submitted work; LG reports personal fees from Grünenthal, during the conduct of the study; DW reports personal fees from Grünenthal, during the conduct of the study, personal fees from AbbVie, BMS, MSD, Pfizer, Roche Chugai, Amgen, Nordic Pharma, UCB, Novartis, Janssen, Celgene, Hospira, Lilly and Sandoz, outside the submitted work, indirect interests: AbbVie, Pfizer, Roche Chugai, MSD, UCB, Mylan, Fresenius Kabi. CD reports personal fees from Grünenthal, during the conduct of the study; A-PT reports personal fees from Menarini, personal fees from Recordati, outside the submitted work.

Patient consent for publication Not required.

Ethics approval No ethics committee was solicited due to the review format of this manuscript, in accordance with the French laws.

Provenance and peer review Not commissioned; externally peer reviewed. Data availability statement All data relevant to the study are included in the article. Open access This is an open access article distributed in accordance with the Creative Commons Attribution Non Commercial (CC BY-NC 4.0) license, which permits others to distribute, remix, adapt, build upon this work non-commercially, and license their derivative works on different terms, provided the original work is properly cited, appropriate credit is given, any changes made indicated, and the use is non-commercial. See: http://creativecommons.org/licenses/by-nc/4.0/.

ORCID iDs

Florian Bailly http://orcid.org/0000-0003-2787-4309

Daniel Wendling http://orcid.org/0000-0002-4687-5780

Anne-Priscille Trouvin http://orcid.org/0000-0002-3524-7455

\section{REFERENCES}

1 Jensen TS, Baron R, Haanpää M, et al. A new definition of neuropathic pain. Pain 2011;152:2204-5.

2 Scholz J, Woolf CJ. The neuropathic pain triad: neurons, immune cells and glia. Nat Neurosci 2007;10:1361-8.

3 Gwak YS, Hulsebosch CE, Leem JW. Neuronal-glial interactions maintain chronic neuropathic pain after spinal cord injury. Neural Plast 2017;2017:1-14.

4 Bouhassira D, Lantéri-Minet M, Attal N, et al. Prevalence of chronic pain with neuropathic characteristics in the general population. Pain 2008;136:380-7.

5 van Hecke O, Austin SK, Khan RA, et al. Neuropathic pain in the general population: a systematic review of epidemiological studies. Pain 2014;155:654-62.

6 Thakur M, Dickenson AH, Baron R. Osteoarthritis pain: nociceptive or neuropathic? Nat Rev Rheumatol 2014;10:374-80.

7 Scholz J, Finnerup NB, Attal N, et al. The IASP classification of chronic pain for ICD-11: chronic neuropathic pain. Pain 2019;160:53-9.

8 Perrot S, Cohen M, Barke A, et al. The IASP classification of chronic pain for ICD-11: chronic secondary musculoskeletal pain. Pain 2019;160:77-82.

9 Petersen KK, Olesen AE, Simonsen O, et al. Mechanistic pain profiling as a tool to predict the efficacy of 3-week nonsteroidal anti-inflammatory drugs plus paracetamol in patients with painful knee osteoarthritis. Pain 2018; Articles in Press.

10 Kurien T, Arendt-Nielsen L, Petersen KK, et al. Preoperative neuropathic pain-like symptoms and central pain mechanisms in knee osteoarthritis predicts poor outcome 6 months after total knee replacement surgery. J Pain 2018;19:1329-41.

11 Haanpää M, Attal N, Backonja M, et al. NeuPSIG guidelines on neuropathic pain assessment. Pain 2011;152:14-27.

12 Bouhassira D, Attal N, Alchaar $\mathrm{H}$, et al. Comparison of pain syndromes associated with nervous or somatic lesions and development of a new neuropathic pain diagnostic questionnaire (DN4). Pain 2005;114:29-36. 
13 Freynhagen R, Baron R, Gockel U, et al. painDETECT: a new screening questionnaire to identify neuropathic components in patients with back pain. Curr Med Res Opin 2006;22:1911-20.

14 Bennett M. The LANSS pain scale: the leeds assessment of neuropathic symptoms and signs. Pain 2001;92:147-57.

15 Bouhassira D, Attal N. Diagnosis and assessment of neuropathic pain: the saga of clinical tools. Pain 2011;152:S74-83.

16 Bouhassira D, Attal N, Fermanian J, et al. Development and validation of the Neuropathic Pain Symptom Inventory. Pain 2004;108:248-57.

17 Finnerup NB, Haroutounian S, Kamerman P, et al. Neuropathic pain: an updated grading system for research and clinical practice. Pain 2016;157:1599-606

18 Koroschetz J, Rehm SE, Gockel U, et al. Fibromyalgia and neuropathic pain - differences and similarities. A comparison of 3057 patients with diabetic painful neuropathy and fibromyalgia. BMC Neurol 2011;11:55

19 Muramatsu K, Tanaka H, Taguchi T. Peripheral neuropathies of the forearm and hand in rheumatoid arthritis: diagnosis and options for treatment. Rheumatol Int 2008;28:951-7.

20 Geoghegan JM, Clark DI, Bainbridge LC, et al. Risk factors in carpal tunnel syndrome. J Hand Surg Br 2004;29:315-20.

21 Omdal R, Loseth S, Torbergsen T, et al. Peripheral neuropathy in systemic lupus erythematosus - a longitudinal study. Acta Neurol Scand 2001;103:386-91.

22 Puéchal X, Said G, Hilliquin P, et al. Peripheral neuropathy with necrotizing vasculitis in rheumatoid arthritis. Arthritis Rheum 1995;38:1618-29.

23 Chai J, Herrmann DN, Stanton M, et al. Painful small-fiber neuropathy in Sjogren syndrome. Neurology 2005;65:925-7.

24 Tavee J, Culver D. Sarcoidosis and small-fiber neuropathy. Current pain and headache reports. 2011;15:201-6.

25 Kosek E, Cohen M, Baron R, et al. Do we need a third mechanistic descriptor for chronic pain states? PAIN 2016;157:1382-6.

26 IASP terminology 2018. Available https://www.iasp-pain.org/Educa tion/Content.aspx?ItemNumber=1698\#Nociplasticpain

27 Latremoliere A, Woolf CJ. Central sensitization: a generator of pain hypersensitivity by central neural plasticity. J Pain 2009;10:895-926.

28 Woolf CJ. Central sensitization: implications for the diagnosis and treatment of pain. Pain 2011;152:S2-15.

29 Wolfe F, Clauw DJ, Fitzcharles M-A, et al. 2016 revisions to the 2010/ 2011 fibromyalgia diagnostic criteria. Semin Arthritis Rheum 2016;46:319-29.

30 Mayer TG, Neblett R, Cohen $\mathrm{H}$, et al. The development and psychometric validation of the Central Sensitization Inventory (CSI). Pain Pract 2012;12:276-85.

31 Neblett R, Cohen H, Choi Y, et al. The Central Sensitization Inventory (CSI): establishing clinically-significant values for identifying central sensitivity syndromes in an outpatient chronic pain sample. J Pain 2013;14:438-45.

32 Scerbo T, Colasurdo J, Dunn S, et al. Measurement properties of the central sensitization inventory: a systematic review. Pain Pract 2018;18:544-54.

33 Soni A, Wanigasekera V, Mezue M, et al. Central sensitization in knee osteoarthritis: relating presurgical brainstem neuroimaging and pain
DETECT - based patient stratification to arthroplasty outcome. Arthritis Rheumatol 2019;71:550-60.

34 Ahmed S, Magan T, Vargas M, et al. Use of the painDETECT tool in rheumatoid arthritis suggests neuropathic and sensitization components in pain reporting. J Pain Res 2014;7:579-88.

35 Rolke R, Baron R, Maier C, et al. Quantitative sensory testing in the German research network on neuropathic pain (DFNS): standardized protocol and reference values. Pain 2006;123:231-43.

36 Rifbjerg-Madsen S, Christensen AW, Christensen R, et al. Pain and pain mechanisms in patients with inflammatory arthritis: a Danish nationwide cross-sectional DANBIO registry survey. PLoS One 2017;12:e0180014.

37 Meeus M, Vervisch S, De Clerck LS, et al. Central sensitization in patients with rheumatoid arthritis: a systematic literature review. Semin Arthritis Rheum 2012;41:556-67.

38 Yesil $\mathrm{H}$, Sungur U, Akdeniz S, et al. Association between serum vitamin $D$ levels and neuropathic pain in rheumatoid arthritis patients: a cross-sectional study. Int J Rheum Dis 2018;21:431-9.

39 Ito S, Kobayashi D, Murasawa A, et al. An analysis of the neuropathic pain components in rheumatoid arthritis patients. Intern Med 2018;57:479-85.

40 Dimitroulas T, Duarte RV, Behura A, et al. Neuropathic pain in osteoarthritis: a review of pathophysiological mechanisms and implications for treatment. Semin Arthritis Rheum 2014;44:145-54.

41 Bannuru RR, Osani MC, Vaysbrot EE, et al. OARSI guidelines for the non-surgical management of knee, hip, and polyarticular osteoarthritis. Osteoarthritis Cartilage 2019;27:1578-89.

42 Yarnitsky D, Crispel Y, Eisenberg E, et al. Prediction of chronic post-operative pain: pre-operative DNIC testing identifies patients at risk. Pain 2008;138:22-8.

43 Kehlet H, Jensen TS, Woolf CJ. Persistent postsurgical pain: risk factors and prevention. Lancet 2006;367:1618-25.

44 Haroutiunian S, Nikolajsen L, Finnerup NB, et al. The neuropathic component in persistent postsurgical pain: a systematic literature review. Pain 2013;154:95-102.

45 Goebel A. Complex regional pain syndrome in adults. Rheumatology 2011;50:1739-50.

46 Freynhagen R, Baron R. The evaluation of neuropathic components in low back pain. Curr Pain Headache Rep 2009;13:185-90.

47 Colloca L, Ludman T, Bouhassira D, et al. Neuropathic pain. Nat Rev Dis Primers 2017;3:17002.

48 Martinez V, Attal N, Bouhassira D, et al. Les douleurs neuropathiques chroniques: diagnostic, évaluation et traitement en médecine ambulatoire. Recommandations pour la pratique clinique de la Société française d'étude et de traitement de la douleur. Douleurs Eval Diagn Trait 2010;11:3-21.

49 Finnerup NB, Attal N, Haroutounian S, et al. Pharmacotherapy for neuropathic pain in adults: a systematic review and meta-analysis. Lancet Neurol 2015;14:162-73.

50 NICE. Neuropathic pain: Pharmacological management. 2013. Available https://www.nice.org.uk/guidance/cg173/evidence/fullguideline-pdf-4840898221

51 Macfarlane GJ, Kronisch C, Dean LE, et al. EULAR revised recommendations for the management of fibromyalgia. Ann Rheum Dis 2017;76:318-28. 